\title{
La congiunzione «e» come elemento introduttore nei titoli giornalistici
}

\author{
Fiammetta Cincera \\ Laura T arricone
}

\begin{abstract}
The purpose of this paper is to describe and in some ways to justify the very frequent use of the conjunction «e» at the beginning of italian newspaper headlines.

0 ur observations are based on the analysis of four widely known contemporary Italian newspapers (using a corpus composed of 30 issues for each newspaper).

An attempt is made to sketch a typology of the functions of this conjunction. The «e» can operate as a relational connector. The interpretation of its function depends on the analysis of cotext (in this case the established link is «endophotic») or of extralinguistic context (in this case the link is «exophoric»). O $n$ the other hand, the «e» can emphasize a particular element in the syntactic structure of the headline.

O ur study is oriented towards a didactic application in intermediate and advanced I talian language lessons. For this reason, in the final section of our paper we propose some examples of exercises.
\end{abstract}

L'idea di svolgere questo studio sull'uso della congiunzione «e» come elemento introduttore nei titoli giornalistici è sorta grazie alla nostra attività didattica in corsi avanzati di lingua italiana. U tilizzando infatti il quotidiano come strumento di lavoro, ci siamo spesso trovate di fronte alla necessità di giustificare la frequente presenza della «e» all'inizio dei titoli giornalistici.

Con il fine di studiare tale fenomeno abbiamo raccolto quotidiani a diffusione nazionale quali La Repubblica (R), II Corriere della Sera (CS), La Stampa (S) ell M essaggero (M ), fino ad ottenere un corpus di 30 numeri per testata dal quale sono stati selezionati gli articoli con titoli introdotti dalla «e».

La «e» è una congiunzione particolarmente ambigua: è polifunzionale e infatti può assumere funzioni diverse identificabili grazie al cotesto 0 al contesto. Spesso la congiunzione si combina con altri elementi che rendono più esplicita la sua funzione (come in: «e quindi» in cui la funzione è conclusiva, «e anche» in cui la funzione è aggiuntiva, «e invece» in cui la funzione è avversativa). ${ }^{1} \mathrm{~N}$ ei titoli da noi analizzati, ci sono esempi che confermano tale ipo- 
tesi. In essi è come se la «e» si unisse al connettivo seguente formando con esso una specie di «collocazione»:

1. «E dopo la partita, a lezione» (R 19/8/95)

2. «E alla finenacque la Lega Roma-Lazio» (CS 19/4/94)

Q uando la «e» viene usata da sola, la sua funzione non è sempre facilmente riconoscibile, specialmente quando tale congiunzione è posta all'inizio di un testo o di un metatesto titolo. 2

Essendo una congiunzione, la «e» è un operatore di coordinazione. Si può parlare di coordinazione solo se si hanno due o più elementi connessi (sintagmaticamente 0 no). ${ }^{3} \mathrm{~N}$ ei titoli introdotti dalla «e» è presente solamente il secondo elemento della coordinazione, il primo deve essere recuperato dal lettore 0 in un articolo precedente o nell'articolo al quale il titolo si riferisce 0 nel contesto extralinguistico. Tuttavia, ci sono anche casi in cui la «e» non è un elemento di connessione ma svolge una funzione di enfasi. Ed infine, in molti casi le due funzioni (di connessione e di enfasi) sembrano essere compresenti.

Pertanto, dall'analisi del nostro corpus ci è sembrato utile mettere a punto una non facile classificazione facendo una prima suddivisione tra «e» come connettivo relazionale ed «e» come operatore di enfasi (tematizzatore).

II connettivo relazionale «e» è un elemento che noi denominiamo «forico», ossia un elemento la cui interpretazione dipende dall'analisi del cotesto (in questo caso il connettivo opera un collegamento endoforico) 0 dall'analisi del contesto (in questo caso il collegamento è esoforico). II rapporto tra gli elementi coordinati dalla «e» può essere:

A) di tipo conclusivo: le proposizioni sono unite da un rapporto di causa/ effetto e l'ultima proposizione rappresenta una conseguenza di quanto espresso precedentemente.

B) di tipo aggiuntivo:

- rispetto ad una o più proposizioni precedenti, la proposizione preceduta dalla «e» rappresenta un'aggiunta di informazione sullo stesso argomento;

- rispetto alla/e proposizione/i precedente/i, la proposizione introdotta dalla «e» rappresenta un evento successivo lungo l'asse temporale.

C) di tipo avversativo: gli elementi coordinati sono presentati in contrapposizione.

2. N onostantela sua struttura generalmente nominale e la sua brevità, il titolo è un testo o più precisamente un «metatesto» in quanto non ha un uno statuto autonomo ma si riferisce ad un al tro testo (nel nostro caso l'articolo giornalistico). C fr. I arovici-Amel (1989: 441-459).

3. Giuliani (1974: 125-139). 
Q uando il collegamento effettuato dalla «e» è endoforico, occorre capire dove si trovi la proposizione a cui fa riferimento il titolo introdotto dalla congiunzione e definire il tipo di connessione che la «e» stabilisce.

O sserviamo il seguente esempio:

\section{3. «E Tomba non sa frenare» (R $9 / 1 / 94)$}

Considerato il cotesto nel quale si trova questo titolo, occorre dire che precedentemente, sulla stessa pagina, era presente un altro articolo (dal titolo: «Belfrond, sembra una favola») al quale il titolo dell'esempio 3 non solo si collegava tematicamente ma stabiliva con esso, mediante la «e», una connessione sintattica di contrapposizione.

La proposizione o le proposizioni alle quali si collega la frase-titolo possono anche essere presenti nello stesso articolo.

\section{4. «E sul cono trionfa un bel gelato al salmone» (S 5/9/95)}

Q ui infatti il titolo non è collegato con gli altri articoli della stessa pagina ma forma con l'articolo al quale si riferisce un corpo a se stante. II titolo è una frase estrapolata dall'articolo: in tal caso la «e» segnala il collegamento (qui di tipo aggiuntivo) di tale frase con altre frasi enunciate nell'articolo. II «gelato al salmone», citato nella frase-titolo, è infatti uno dei tanti gusti stravaganti di cui si parla in varie frasi presenti nel testo.

$\mathrm{N}$ el caso del collegamento esoforico, invece, il rapporto di connessione viene stabilito non con una proposizione espressa esplicitamente ma con un insieme di informazioni sullo stesso argomento derivate dalle conoscenze che il lettore dovrebbe avere sulla real tà extralinguistica e che sono sottintese dal giornalista. Ciò è abbastanza frequente nel discorso giornalistico, in quanto esso non è discorso individuale dell'autore ma un discorso collettivo che coinvolge la memoria (a breve o a lungo termine) di un'intera comunità linguistica. II lettore pertanto deve cercare il primo elemento della coordinazione in questo bagaglio di conoscenze previe. ${ }^{4} 0$ sserviamo il seguente esempio:

5. «E l'Europa sale a quindici» (R 2/3/94)

4. Le frasi che costituiscono i titoli giornalistici hanno degli scopi che il destinatario deve ricostruire. Tali scopi sono:

- scopi immediati: vengono ricostruiti dal lettore utilizzando la competenza linguistica (le sue conoscenze del vocabolario e della grammatica della lingua usata nel brano);

- sovrascopi : vengono ricostruiti facendo ricorso alla competenza extralinguistica. II lettore ricostruisce i sovrascopi, attraverso processi di inferenza che vengono attivati in base alle conoscenze che possiede riguardo al mondo, al contesto della situazione, alla cosiddetta encidopedia di conoscenze. (Parisi, 1979: 95-123) 
Q uesto titolo, che appare in prima pagina, introduce il risultato di una serie di trattative relative all'annessione di tre paesi alla comunità europea, trattative che il giornalista presuppone siano già conosciute dal lettore. Q uindi, il rapporto che qui la «e» stabilisce tra le conoscenze del lettore e la frase-titolo è di tipo conclusivo.

Secondo quanto detto prima, la congiunzione «e», oltre ad essere un connettivo relazionale, può mettere in rilievo un elemento del titolo agendo in questo modo come operatore di enfasi. La «e» in questi casi «marca» sintatticamente la struttura del titolo. L'elemento costituente che segue la congiunzione rappresenta il tema del titolo e spesso dell'intero articolo (in quest'ultimo caso il tema è anche un macrotema). ${ }^{5}$

L'italiano è una lingua il cui ordine sintattico non marcato presenta il soggetto in prima posizione seguito dal verbo ed eventualmente da complementi di diverso tipo. In questi casi, il soggetto (che può rappresentare l'informazione data o nuova) costituisce anche il tema. La «e» può però segnalare anche la tematizzazione di un costituente diverso dal soggetto (di solito un complemento di luogo o di tempo). Gli elementi che più spesso vengono enfatizzati sono infatti rappresentati da:

a) Costituenti nominali nucleari con funzione di soggetto (di solito sintagmi nominali determinati o nomi propri):

\section{6. «E Faenza cancella Piazza Pietro N enni» (CS 28/7/95)}

b) Espressioni di luogo (avverbi o sintagmi preposizionali):

7. «E dalla curva un solo grido, andate a lavorare» (R 31/3/95)

8. «E in passerella sfilano le diavolesse» ( $R 3 / 3 / 94)$

9. «E al Parma ci va Casiraghi» (M 15/6/95)

c) Avverbi di tempo (specialmente avverbi deittici come «ora» e «adesso» che attualizzano la notizia):

10. «E oggi arrivano i calendari» (S $1 / 8 / 95)$

11. «E ora in Germania il calcio fa boom» (S 7/8/95)

N ei titoli sopra citati, la «e» non è un elemento sintatticamente necessario ma rende solamente più esplicita l'articolazione del titolo in due parti. In questi casi la «e» può essere eliminata senza che ciò produca un cambiamento sostanziale del significato del titolo.

Sembrano estremamente interessanti i due casi esemplificati in 12 e 13.

12. «E il M arc'Aurelio rinasce sotto il segno di Fellini» (M 25/6/95)

13. «E i giapponesi vedranno in TV come hanno votato gli italiani» (M 29/3/94) 
II tema viene espresso e enfatizzato per richiamare alla memoria del lettore un argomento già conosciuto (ma «nuovo») o per sottolineare un elemento di sorpresa contrario alle aspettative del lettore. $\mathrm{N}$ el titolo esemplificato in 12 , si richiama alla memoria del lettore il tema (che costituisce un'informazione conosciuta ma che al momento della lettura dell'articolo non è presente attivamente nella sua memoria) e si forniscono informazioni nuove rispetto a tale tema. N ell'esempio 13, il tema enfatizzato dalla «e» costituisce per il lettore una sorpresa, infatti non ci si aspetterebbe che i risultati delle nostre elezioni possano arrivare perfino in G iappone.

Ci sono, infine, casi in cui la «e» è solamente una struttura «eco» di titoli o frasi ben conosciuti appartenenti soprattutto alla tradizione letteraria. Tali riferimenti non richiedono la conoscenza dell'opera citata. Infatti, si tratta quasi sempre di un uso preconfezionato di una struttura presente nel bagaglio culturale del lettore e dell'autore. A sostegno di ciò, il titolo giornalistico non presenta quasi mai elementi (virgolette, corsivo, ecc... ) che ci segnalino la presenza di una citazione. II riferimento (p. es. la ripresa di un titolo di un'opera letteraria) viene spesso manipolato dal giornalista, il quale sopprime degli elementi linguistici per aggiungerne degli altri, oppure mantiene gli stessi significanti cambiandone il significato. $6 \mathrm{~A}$ tale proposito riportiamo i seguenti titoli presenti nel nostro corpus:

\section{4. «E i G randi stanno a guardare» (R 24/1/94)}

15. «E le stelle stanno a giocare» (M 28/7/95)

16. «E le stelle fanno shopping» (C S 15/9/95)

Q ui si fa chiaramente riferimento al titolo del romanzo di Cronin E le stelle stanno a guardare o più probabilmente allo sceneggiato televisivo tratto dall'omonimo romanzo. $\mathrm{N}$ el primo caso si riprende l'intero sintagma verbale mentre cambia il sintagma nominale in posizione di soggetto. $\mathrm{N}$ el secondo caso cambia solamente il verbo principale e nel terzo cambia il sintagma verbale. In tutti e tre si mantiene però l'elemento introduttore «e». In nessuno dei casi il riferimento è al contenuto del romanzo ma il titolo viene usato come struttura assimilata nel corso del tempo e indipendente dall'opera. I tre titoli giornalistici ricorrono in modo diverso al titolo di quest'opera letteraria: nel primo caso si allude all'inoperosità delle superpotenze di fronte al conflitto nella ex-I ugoslavia, che ricorda l'inoperosità delle stelle (cielo) di fronte alla successione di avvenimenti, spesso molto tristi, sui quali si basa il romanzo di Cronin; nel secondo e nel terzo caso invece il titolo viene usato riecheggiandone parzialmente il significante ma non il significato.

U n'ultima importante considerazione da fare riguarda l'origine di tale tipo di struttura. Sembra che l'uso della «e» come elemento introduttore nei titoli giornalistici richiami, proprio come «calco sintattico», una struttura 
analoga presente nel titolo o nel primo verso di componimenti poetici appartenenti alla tradizione letteraria dell'O ttocento e del N ovecento.

In questi casi la «e» viene interpretata come elemento di connessione tra il «non detto» e il «detto» e suggerisce un'idea di continuità nella riflessione, nel discorso interiore del poeta.

Se si analizzano i componimenti introdotti dalla «e», si noterà che a volte la congiunzione assume anche funzione di connessione sintattica: ad esempio nel caso della famosa poesia di Q uasimodo $\mathrm{Ed}$ è subito sera il titolo riprende l'ultimo verso del componimento dove la «e» ha anche un valore testuale di tipo conclusivo. ${ }^{7}$

\section{Parte didattica}

L'analisi della «e» va introdotta da una riflessione più ampia sull'uso della lingua dei giornali per studenti di un corso di livello avanzato chesi presuppone possiedano una buona competenza linguistica e discrete conoscenze sulla real tà politica, sociale e culturale italiana.

0 gni esercizio è stato concepito per raggiungere obiettivi specifici e la totalità degli esercizi permetterà allo studente di procedere gradualmente in un percorso di apprendimento finalizzato all'acquisizione di una competenza linguistica globale.

La prima delle due sezioni in cui abbiamo suddiviso gli esercizi è introduttiva e propedeutica e propone esercizi mirati ad un'analisi più ampia del titolo giornalistico, posto che l'identificazione della funzione della «e» iniziale non può prescindere da una comprensione dell'intero titolo; la seconda sezione propone invece esercizi specifici sull'uso della «e».

Il primo gruppo comprende:

a) Esercizi basati sull'analisi di figure retoriche e riferimenti enciclopedici che intendono stimolare la capacità cognitiva dello studente permettendogli di arrivare, attraverso l'uso e l'arricchimento del suo bagaglio di conoscenze enciclopediche, ad una più facile decodificazione del contenuto del titolo.

7. Riportiamo alcuni esempi:

S. Q uasimodo Ed èsubito sera

Alle fronde dei salici: E come potevamo noi cantare [... ]

G. Pascoli II gel somino notturno: E s'aprono i fiori notturni [... ]

$\mathrm{N}$ ella nebbia: E guardai nella valle: era sparito tutto! Sommerso! Era un gran mare piano $[\ldots]$

G. U ngaretti Allegria di naufragi: E subito riprende il viaggio [... ]

La madre: E il cuore quando d'un ultimo battito avrà fatto cadere il muro d'ombra [... ]

Sentimento del tempo: E per la luce giusta cadendo solo un'ombra [... ]

V. Cardarelli $E$ ora, in queste mattine. 
b) Esercizi basati sull'analisi della struttura sintattica del titolo: analisi di strutture ellittiche, strutture nominali, meccanismi di focalizzazione di elementi, di demarcazione del tema e del rema. Tali esercizi mirano a sviluppare la capacità di fare inferenze e di capire quali sono le strategie che l'autore utilizza per guidare il lettore nella comprensione del messaggio.

O ffriamo qui di seguito l'impostazione degli esercizi che consigliamo di proporre e svolgere in classe sull'argomento. Q uesti esercizi andrebbero correlati da titoli ed articoli giornalistici accompagnati dal cotesto (ossia la pagina all'interno della quale compaiono), che per ragioni di spazio non possiamo fornire nella presente pubblicazione.

\section{Esercizi della prima sezione:}

Esercizio1: identificare le figure retoriche presenti nei titoli espiegarle.

Tale esercizio mira a sviluppare la capacità di riconoscere al cune figure retoriche frequentemente usate per fare riferimento a situazioni politiche 0 sociali.

Esercizio 2: dopo aver letto l'articolo, introdurre il verbo mancante nei titoli con ellissi.

L'obiettivo è quello di sviluppare le capacità del discente di ricostruire le parti mancanti attraverso le sue conoscenze lessicali e grammaticali.

Esercizio 3: riformulare i titoli secondo un ordine non marcato e avanzare delle ipotesi sulle ragioni che hanno indotto il giornalista ad ordinare e a strutturare in tal modo gli elementi del titolo.

L'obiettivo è far riflettere sulle informazioni supplementari date dall'ordine dei sintagmi.

\section{Esercizi della seconda sezione:}

Esercizio 1: indicare se con il titolo si riassume il contenuto dell'articolo o se ne estrapola una singola informazione, centrale o periferica.

In questo modo si mira a ricostruire i processi di codificazione di un titolo, utili anche per comprendere la funzione della «e».

Esercizio 2:

a) $\mathrm{N}$ ei casi in cui la «e» collega il titolo con un altro titolo/articolo presente nella stessa pagina, definire il tipo di relazione che la congiunzione stabilisce.

b) N ei casi in cui la «e» rimanda a conoscenze enciclopediche, individuare i riferimenti extralinguistici.

L'obiettivo di questo esercizio è far recuperare nel cotesto o nel contesto il primo elemento della struttura coordinata.

Esercizio 3: formulare un titolo con la «e» partendo dall'articolo e dal cotesto dati. 


\section{Bibliografia essenziale}

BAzZAN ELLA, C. (1985). «L'uso dei connettivi nel parlato: alcune proposte». In Franchi de Bellis-Savoia: 83-94.

Berretta, M. (1984). «C onnettivi testuali in italiano e pianificazione del discorso». In Coveri: 237-254.

CASADEI, E. (1980). «C ontributi per una teoria del titolo». Lingua estile, XV/1: 3-25.

CECCHINI, M . (1985). «Alcune caratteristiche di struttura informativa del linguaggio giornalistico». In Franchi de Bellis-Savoia: 33-43.

CoverI, L. (1984) (a cura di). Linguistica testuale (Atti del XVI congresso internazionaleSLI). Roma: Bulzoni.

Di Fazio Alberti, M. (1985). «I titolo dell'opera letteraria nella lingua dei giornali». Cultura escuola, 96: 14-33.

EsCAN DELL VIDAL, M .V . (1993). Introducción a la pragmática. Barcellona: Antropos.

Fran ChI de Bellis, A.; Savoia, L.M . (1985) (a cura di), Sintassi e M orfologia della Lingua Italiana d'uso. Teorie ed Applicazioni Descrittive (Atti del XVII congresso internazionale SLI). Roma: Bulzoni.

GIULIANI, M. V. (1974). «La coordinazione in italiano: e». In M edici-Sangregorio: 125-139.

IAROVICI, E.; AmEL, R. (1989). «The strategy of the headline». Semiotica, 77/4: 441-459.

Medicl, M .; SAngregorio, A. (1974) (a cura di). Fenomeni morfologid e sintattici nell'italiano contemporaneo, (Atti del VI congresso internazionale SLI). Roma: Bulzoni.

PARISI, D . (1979). Per un'educazionelinguistica razionale. Bologna: II M ulino.

RenzI, L. et alii (1995) (a cura di). Grande grammatica italiana di consultazione, vol. III. Bologna: II M ulino.

Rocher TANUGI, J. (1974). «M odéles syntaxiques dans les titres de journaux et les slogans». In M edici-Sangregorio: 475-490.

SABAtinI, F. (1984). La comunicazione e gli usi della lingua. Torino: Loescher.

Sen SIn I, M .; SeRIAn NI, L. (1991). Grammatica Italiana. T orino: UTET. 